\title{
COMMENTARY
}

\section{Further cautions for the use of ventilatory-induced changes in arterial pressures to predict volume responsiveness}

\author{
Sheldon Magder* \\ See related research by Wyler et al., http://ccforum.com/content/14/3/R111, and related research by Daudel et al., \\ http://ccforum.com/content/14/3/R122
}

\begin{abstract}
Variations in systemic arterial pressure with positivepressure breathing are frequently used to guide fluid management in hemodynamically unstable patients. However, because of the complex physiology that determines the response, there are important limitations to their use. Two papers in a previous volume add pulmonary hypertension as limitations. Uncritical use of ventilatory-induced changes in arterial pressure can lead to excessive volume therapy and potential clinical harm, and they must be used with respect and thought.
\end{abstract}

Ventilatory-induced variations in arterial pulse pressure (PPV) are widely used to predict whether a patient is volume responsive, but they have important limitations. Wyler and colleagues add pulmonary hypertension as another limitation [1]. The authors should be commended for not stopping with their clinical observation, confirming this in an animal model that - although somewhat different from the clinical condition - allowed controlled conditions [2]. Ventilatory variations in arterial pressure were proposed over 20 years ago [3] and algorithms for their use are now included in a number of monitoring devices. Important to remember, however, is that these indicators are only useful if prerequisites are met - including the absence of any spontaneous ventilatory efforts, a regular rhythm, and ventilatory settings similar to those in the original studies. The current studies add another limitation and importantly indicate that indiscriminant use of these indicators can lead to excessive fluid use.

*Correspondence: sheldon.magder@muhc.mcgill.ca

Royal Victoria Hospital, 687 Pine Avenue West, Montreal, Quebec, Canada H3A 1A1
I have argued previously [4] - and still believe - that the dominant process causing ventilatory-induced fluctuations in arterial pressure that are fluid responsive is that when the heart is functioning on the steep volumeresponsive part of the cardiac function curve, the inspiratory rise in pleural pressure transiently decreases return of blood to the right heart. This decrease in flow is passed to the left side of the circulation during expiration. When the heart is functioning on the flat nonvolumeresponsive part of the cardiac function curve, a fall in cardiac filling is less marked. This mechanism dominates because the pressure gradient from the large systemic venous reservoir to the right heart is only 4 to $8 \mathrm{mmHg}$ so small changes in pleural pressure can have a major effect on venous return.

Since the normal gradient for venous return is small, even small increases in pleural pressure might be expected to reduce cardiac output to zero - yet observed decreases in pulse pressure and stroke volume are much more modest. This observation occurs because pulmonary blood volume provides a reserve that can temporarily maintain left-sided cardiac filling. The volume in the pulmonary vasculature, the respiratory rate, and the heart rate determine the magnitude of this buffering effect.

During inspiration, lung inflation also squeezes volume from the pulmonary veins and decreases left ventricular afterload [5-7]. These two factors produce a transient increase in left ventricular ejection, and account for the inspiratory increase in pressure relative to the value at end-expiration (dUp) in arterial pressure variations [4], but this component has little volume sensitivity. This lack of sensitivity is because the thoracic vascular compliance is only one-seventh that of the systemic vascular compliance and a change in total body volume adds only a small amount of volume to this compartment. Yet this small volume, when transferred to the arterial side, has a large pressure effect because of the low arterial compliance. 
There are other mechanisms that can produce PPV with positive pressure ventilation. Veiellard-Baron and coworkers [8] showed that inspiratory loading can significantly reduce right ventricular output. This can be explained as follows. When the lung is in West Zone III, lung inflation produces a negligible load on the right ventricle [5]; but when it is in West Zone II, lung inflation can markedly decrease right ventricular output, increase pulmonary vascular volume and transiently decrease left ventricular filling [9]. The consequent decrease in left ventricular output can produce very large swings in arterial pressures, but these swings should be minimally responsive to volume infusion because they are minimally related to right heart filling.

Based on the above analysis, how can the poor predictive values of PPV in the studies by Wyler and colleagues [1] and by Daudel and colleagues [2] be explained? Their plots of stroke volume against central venous pressure indicate that stroke volume was responsive at some point even in the endotoxin group and there was a lot more volume responsiveness than seems to show up in the results. One factor could be simply technical. The authors used the standard 10\% change in stroke volume. After hemorrhage this would mean a change in stroke volume of only 1 to $2 \mathrm{ml}$ versus $10 \mathrm{ml}$ in the control animals at their peak. Yet a $1 \mathrm{ml}$ change in end-diastolic volume from any initial value should produce a $1 \mathrm{ml}$ change in stroke volume. The use of percentage change could thus have obscured what was happening, especially considering that there were progressive increases in the stroke volumes.

Two other factors also might be involved. First, dUp probably accounted for a significant part of the PPV. dUp is related to the decrease in afterload with a positive pressure breath and the squeezing of blood out of the lungs. Afterload reduction has a greater effect when ventricular function is decreased, as in sepsis; and, secondly, more volume can be squeezed from the lung if pulmonary blood volume was increased in the septic animals. Furthermore, the afterload reducing effect is related to how much pleural pressure rises with each breath, and pleural pressure would have been increased if chest wall compliance was reduced by edema from volume loading. Second, lung injury associated with sepsis probably increased the presence of zone II conditions in the lungs, so this cause of PPV is not volume responsive.

These studies further emphasize the limited usefulness of ventilatory-induced changes in arterial pressure for predicting volume responsiveness. There are so many factors that can affect the phenomena that the technique's use should be reserved for very limited controlled conditions such as in the operating room. The authors' warning about potential harm from excess use of fluids if these measurements are used too casually needs to be heeded. Finally, it is always worth emphasizing that even if PPV does predict volume responsiveness, it does not mean that the patient actually needs volume or that volume is the best management choice.

\section{Abbreviations}

dUP, inspiratory increase in pressure relative to value at end-expiration; PPV, pulse pressure variation.

\section{Competing interests}

The author declares that he has no competing interests.

Published: 20 September 2010

\section{References}

1. Wyler MvB, Takala J, Roeck M, Porta F, Tueller D, Ganter CC, Schröder R, Bracht $H$, Baenziger $B$, Jakob SM: Pulse-pressure variation and hemodynamic response in patients with elevated pulmonary artery pressure: a clinical study. Crit Care 2010, 14:R111.

2. Daudel F, Tueller D, Krahenbuhl S, Jakob SM, Takala J: Pulse pressure variation and volume responsiveness during acutely increased pulmonary artery pressure: an experimental study. Crit Care 2010, 14:R122.

3. Perel A, Pizov R, Cotev S: Systolic blood pressure variation is a sensitive indicator of hypovelemia in ventilated dogs subjected to graded hemorrhage. Anesthesiology 1987, 67:498-502.

4. Magder S: Clinical usefulness of respiratory variations in arterial pressure. Am J Respir Crit Care Med 2004, 169:151-155.

5. Permutt S, Howell JBL, Proctor DF, Riley RL: Effect of lung inflation on static pressure volume characteristics of pulmonary vessels. J Appl Physiol 1961, 16:64-70.

6. Bromberger-Barnea B: Mechanical effects of inspiration on heart functions. Fed Proc 1981, 40:2172-2177.

7. Howell JB, Permutt S, Proctor DF, Riley RL: Effect of inflation of the lung on different parts of pulmonary vascular bed. J App/ Physio/ 1961, 16:71-76.

8. Vieillard-Baron A, Prin S, Chergui K, Dubourg O, Jardin F: Echo-Doppler demonstration of acute cor pulmonale at the bedside in the medical intensive care unit. Am J Respir Crit Care Med 2002, 166:1310-1319.

9. Permutt S, Bromberger-Barnea B, Bane HN: Alveolar pressure, pulmonary venous pressure, and the vascular waterfall. Med Thorac 1962, 19:239-260.

doi:10.1186/cc9223

Cite this article as: Magder S: Further cautions for the use of ventilatoryinduced changes in arterial pressures to predict volume responsiveness. Critical Care 2010, 14:197. 